\title{
MONOTONICITY RESULT FOR GENERALIZED LOGARITHMIC MEANS
}

\author{
XIN LI, CH.-P. CHEN AND F. QI
}

Abstract. $r \mapsto \frac{L_{r}(a, b)}{L_{r}(1-a, 1-b)}$ is a strictly increasing function of $r \in(-\infty, \infty)$ for $0<a<b \leq \frac{1}{2}$, and is a strictly decreasing function of $r \in(-\infty, \infty)$ for $\frac{1}{2} \leq a<b<1$, where $L_{r}(a, b)$ denotes the generalized logarithmic mean of two positive numbers $a$ and $b$.

\section{Introduction}

The following inequality in [1, p. 5] is due to Ky Fan: If $0<x_{i} \leq \frac{1}{2}$ for $i=1,2, \ldots, n$, then

$$
\left(\frac{\prod_{i=1}^{n} x_{i}}{\prod_{i=1}^{n}\left(1-x_{i}\right)}\right)^{1 / n} \leq \frac{\sum_{i=1}^{n} x_{i}}{\sum_{i=1}^{n}\left(1-x_{i}\right)}
$$

with equality only if all the $x_{i}$ are equal.

Inequality (11) can be written as

$$
\frac{M_{0}(x)}{M_{0}(1-x)} \leq \frac{M_{1}(x)}{M_{1}(1-x)}
$$

where $M_{r}(x)$ denotes the $r$-order power mean of $x_{i}>0$ for $i=1,2, \ldots, n$, defined by

$$
M_{r}(x)= \begin{cases}\left(\frac{1}{n} \sum_{i=1}^{n} x_{i}^{r}\right)^{1 / r}, & r \neq 0 ; \\ \left(\prod_{i=1}^{n} x_{i}\right)^{1 / n}, & r=0 .\end{cases}
$$

Zh. Wang, J. Chen and X. Li 12] found the necessary and sufficient condition for

$$
\frac{M_{r}(x)}{M_{r}(1-x)} \leq \frac{M_{s}(x)}{M_{s}(1-x)}
$$

when $r<s$.

Received March 2, 2005; revised September 6, 2005.

2000 Mathematics Subject Classification. 26A48, 26D20.

Key words and phrases. Generalized logarithmic mean, monotonicity, Ky Fan inequality.

Supported in part by the Science Foundation of the Project for Fostering Innovation Talents at Universities of Henan Province, China, NSF of Henan Province (0511012000). 
In 1975, Stolarsky [10] defined the extended means $E(r, s ; x, y)$ by

$$
\begin{array}{lll}
E(r, s ; x, y)=\left(\frac{r}{s} \cdot \frac{y^{s}-x^{s}}{y^{r}-x^{r}}\right)^{1 /(s-r)}, & & r s(r-s)(x-y) \neq 0 ; \\
E(r, 0 ; x, y)=\left(\frac{1}{r} \cdot \frac{y^{r}-x^{r}}{\ln y-\ln x}\right)^{1 / r}, & & r(x-y) \neq 0 ; \\
E(r, r ; x, y)=\frac{1}{e^{1 / r}}\left(\frac{x^{x^{r}}}{y^{y^{r}}}\right)^{1 /\left(x^{r}-y^{r}\right)}, & & r(x-y) \neq 0 ; \\
E(0,0 ; x, y)=\sqrt{x y}, & & x \neq y ; \\
E(r, s ; x, x)=x, & & x=y .
\end{array}
$$

It is known that $E(r, s ; x, y)$ are increasing with both $r$ and $s$, or with both $x$ and $y$ (see [2, 4, 10]). A comparison theorem for the extended means has been obtained by E. B. Leach and M. C. Sholander in [5]. In [9], the logarithmic convexity of $E$ was proved.

Taking in $E(r, s ; x, y) r=1$ and $s=r+1$, we obtain the generalized logarithmic mean $L_{r}(a, b)$ of two positive numbers $a, b$ : For $a=b$ by $L_{r}(a, b)=a$ and for $a \neq b$ by

$$
\begin{aligned}
L_{r}(a, b) & =\left(\frac{b^{r+1}-a^{r+1}}{(r+1)(b-a)}\right)^{1 / r}, \quad r \neq-1,0 ; \\
L_{-1}(a, b) & =\frac{b-a}{\ln b-\ln a}=L(a, b) \\
L_{0}(a, b) & =\frac{1}{e}\left(\frac{b^{b}}{a^{a}}\right)^{1 /(b-a)}=I(a, b),
\end{aligned}
$$

where $L(a, b)$ and $I(a, b)$ are respectively the logarithmic mean and the exponential mean of two positive numbers $a$ and $b$. When $a \neq b, L_{r}(a, b)$ is a strictly increasing function of $r$. In particular,

$$
\begin{aligned}
\lim _{r \rightarrow-\infty} L_{r}(a, b) & =\min \{a, b\}, & \lim _{r \rightarrow+\infty} L_{r}(a, b) & =\max \{a, b\}, \\
L_{1}(a, b) & =A(a, b), & L_{-2}(a, b) & =G(a, b),
\end{aligned}
$$

where $A(a, b)$ and $G(a, b)$ are the arithmetic and the geometric means, respectively. For $a \neq b$, the following well known inequality holds:

$$
G(a, b)<L(a, b)<I(a, b)<A(a, b) .
$$

In this short note, motivated by inequality (4), we will establish the following.

Theorem 1. $r \mapsto \frac{L_{r}(a, b)}{L_{r}(1-a, 1-b)}$ is a strictly increasing function of $r \in(-\infty, \infty)$ for $0<a<b \leq \frac{1}{2}$, and is a strictly decreasing function of $r \in(-\infty, \infty)$ for $\frac{1}{2} \leq a<b<1$.

As a consequence of Theorem 1, we have 
Corollary 1. If $0<a<b \leq \frac{1}{2}$, then

$$
\begin{aligned}
\frac{a}{1-b}<\frac{G(a, b)}{G(1-a, 1-b)} & <\frac{L(a, b)}{L(1-a, 1-b)} \\
& <\frac{I(a, b)}{I(1-a, 1-b)}<\frac{A(a, b)}{A(1-a, 1-b)}<\frac{b}{1-a} .
\end{aligned}
$$

If $\frac{1}{2} \leq a<b<1$, then (14) is reversed.

\section{Proof of Theorem 1}

In order to verify Theorem 1, we shall make use of the following elementary lemma which can be found in [3, p.395].

Lemma 1.(3, p.395]) Let the second derivative of $\phi(x)$ be continuous with $x \in$ $(-\infty, \infty)$ and $\phi(0)=0$. Define

$$
g(x)=\left\{\begin{array}{cc}
\frac{\phi(x)}{x}, & x \neq 0 \\
\phi^{\prime}(0), & x=0 .
\end{array}\right.
$$

Then $\phi(x)$ is strictly convex (concave) if and only if $g(x)$ is strictly increasing (decreasing) with $x \in(-\infty, \infty)$.

Remark 1. In [7, p. 18] a general conclusion was given: A function $f$ is convex on $[a, b]$ if and only if $\frac{f(x)-f\left(x_{0}\right)}{x-x_{0}}$ is nondecreasing on $[a, b]$ for every point $x_{0} \in[a, b]$.

Proof of Theorem 1. Define for $r \in(-\infty, \infty)$,

$$
\varphi(r)= \begin{cases}\ln \left(\frac{b^{r+1}-a^{r+1}}{(1-a)^{r+1}-(1-b)^{r+1}}\right), & r \neq-1 ; \\ \ln \left(\frac{\ln (b / a)}{\ln [(1-a) /(1-b)]}\right), & r=-1 .\end{cases}
$$

Then

$$
\ln f(r)= \begin{cases}\frac{\varphi(r)}{r}, & r \neq 0 \\ \varphi^{\prime}(0), & r=0 .\end{cases}
$$

In order to prove that $\ln f$ is strictly increasing (decreasing) it suffices to show that $\varphi$ is strictly convex (concave) on $(-\infty, \infty)$. Computation reveals that

$$
\varphi(-1-r)=\varphi(-1+r)+r \ln \frac{(1-a)(1-b)}{a b},
$$

which implies that $\varphi^{\prime \prime}(-1-r)=\varphi^{\prime \prime}(-1+r)$, and then $\varphi$ has the same convexity (concavity) on both $(-\infty,-1)$ and $(-1, \infty)$. Hence, it is sufficient to prove that $\varphi$ is strictly convex (concave) on $(-1, \infty)$. 
A computation yields

$$
\begin{gathered}
\varphi^{\prime}(r)=\frac{b^{r+1} \ln b-a^{r+1} \ln a}{b^{r+1}-a^{r+1}}-\frac{(1-b)^{r+1} \ln (1-b)-(1-a)^{r+1} \ln (1-a)}{(1-b)^{r+1}-(1-a)^{r+1}}, \\
(r+1)^{2} \varphi^{\prime \prime}(r)=(r+1)^{2}\left[-\frac{a^{r+1} b^{r+1}\left(\ln \frac{a}{b}\right)^{2}}{\left(b^{r+1}-a^{r+1}\right)^{2}}+\frac{(1-a)^{r+1}(1-b)^{r+1}\left(\ln \frac{1-b}{1-a}\right)^{2}}{\left[(1-a)^{r+1}-(1-b)^{r+1}\right]^{2}}\right] \\
=-\frac{\left(\frac{a}{b}\right)^{r+1}\left[\ln \left(\frac{a}{b}\right)^{r+1}\right]^{2}}{\left[1-\left(\frac{a}{b}\right)^{r+1}\right]^{2}}+\frac{\left(\frac{1-b}{1-a}\right)^{r+1}\left[\ln \left(\frac{1-b}{1-a}\right)^{r+1}\right]^{2}}{\left[1-\left(\frac{1-b}{1-a}\right)^{r+1}\right]^{2}} .
\end{gathered}
$$

Define for $0<t<1$,

$$
\omega(t)=\frac{t(\ln t)^{2}}{(1-t)^{2}}
$$

Differentiation yields

$$
(1-t) t \ln t \frac{\omega^{\prime}(t)}{\omega(t)}=(1+t) \ln t+2(1-t)=-\sum_{n=2}^{\infty} \frac{n-1}{n(n+1)}(1-t)^{n+1}<0
$$

which implies that $\omega^{\prime}(t)>0$ for $0<t<1$. It is easy to see that

$$
\begin{aligned}
& 0<\left(\frac{a}{b}\right)^{r+1}<\left(\frac{1-b}{1-a}\right)^{r+1}<1 \text { for } 0<a<b \leq \frac{1}{2}, r>-1, \\
& 0<\left(\frac{1-b}{1-a}\right)^{r+1}<\left(\frac{a}{b}\right)^{r+1}<1 \text { for } \frac{1}{2} \leq a<b<1, r>-1,
\end{aligned}
$$

and therefore $\varphi^{\prime \prime}(r)>0$ for $0<a<b \leq \frac{1}{2}$ and $r>-1$, while $\varphi^{\prime \prime}(r)<0$ for $\frac{1}{2} \leq a<b<1$ and $r>-1$. Thus $\varphi$ is strictly convex (concave) on $(-1, \infty)$ for $0<a<b \leq \frac{1}{2}\left(\frac{1}{2} \leq a<\right.$ $b<1)$. The proof is complete.

For various proposed multivariable extensions of the means $E(r, s ; x, y)$ to several variables, see [4, 6, 8,11$]$.

In view of Theorem 1, it is natural to pose the following open problem.

Open Problem. Generalize Theorem 1 to several variables.

\section{Acknowledgements}

The author is indebted to the anonymous referee for many valuable comments and corrections in language expressions.

\section{References}

[1] E. F. Beckenbach and R. Bellman, Inequalities, Springer Verlag, 1961. 
[2] Chao-Ping Chen and Feng Qi, An alternative proof of monotonicity for the extended mean values, Aust. J. Math. Anal. Appl. 1 (2004), no.2, Article 11. Available online at http://ajmaa.org/volumes.php

[3] J.-Ch. Kuang, Applied Inequalities, 2nd ed., Hunan Education Press, Changsha, China, 1993. (Chinese)

[4] E. B. Leach and M. C. Sholander, Extended mean values, Amer. Math. Monthly 85(1978), 84-90.

[5] E. B. Leach and M. C. Sholander, Multi-variable extended mean values, J. Math. Anal. Appl. 104(1984), 390-407.

[6] J. K. Merikowski, Extending means of two variables to several variables, J. Ineq. Pure. Appl. Math. 5(2004), no. 3, Article 65. Available online at http://jipam.vu.edu.au/article.php?sid=411

[7] D. S. Mitrinović, Analytic Inequalities, Springer-Verlag, Berlin, 1970.

[8] J. Pečarić and V. šimić, The Stolarsky-Tobey mean in $n$ variables, Math. Inequal. Appl. 2 (1999), 325-341.

[9] F. Qi, Logarithmic convexity of extended mean values, Proc. Amer. Math. Soc. 130 (2002), 1787-1796, (electronic).

[10] K. B. Stolarsky, Generalizations of the logarithmic mean, Math. Mag. 48(1975), 87-92.

[11] M. D. Tobey, A two-parameter homogeneous mean value, Amer. Math. Monthly 87(1980), 545-548. Proc. Amer. Math. Soc. 18 (1967), 9-14.

[12] Zh. Wang, J. Chen and X. Li, A generalization of the Ky Fan inequality, Univ. Beograd. Publ. Elektrotehn. Fak. 7(1996), 9-17.

College of Mathematics and Informatics, Henan Polytechnic University, Jiaozuo City, Henan 454010, China.

E-mail: lixinxxren@sohu.com

College of Mathematics and Informatics, Research Institute of Applied Mathematics, Henan Polytechnic University, Jiaozuo City, Henan 454010, China.

E-mail: chenchaoping@hpu.edu.cn

Research Institute of Mathematical Sciences, Henan Polytechnic University, Jiaozuo City, Henan 454010, China.

E-mail: qifeng@hpu.edu.cn 
